\title{
DAMPING OF VIBRATION IN ADAPTIVE STRUCTURES - COMPUTER SIMULATION AND EXPERIMENTAL VERIFICATION
}

\author{
Jan Holnicki-Szulc and Zbigniew Marzec \\ Institute of Fundamental Technological Research, \\ Świętokrzyska 21,00-049 Warsaw, Poland, e-mail:holnicki@ippt.gov.pl
}

\begin{abstract}
A new concept of semi-active damping of vibration in adaptive structures (equipped with controllable devices able to switch on and off specially designed structural inter-connections) is presented. Computer simulations as well as experimental tests of performance of self-controlled adaptive structures are demonstrated. High efficiency and easy applicability of the proposed control strategy (based on the simple law realising structural inter-connections for an instant, just after the local vibration pick) is commented.
\end{abstract}

Keywords: damping of vibration, semi-active control, adaptive structures, computer simulation, experimental verification

\section{INTRODUCTION}

The goal of this paper is to discuss a strategy of an impulse release of strain energy for damping of free vibration in adaptive structures (equipped with actively controlled dissipaters). This concept can be applied to skeletal structures as well two-layer beams or plates, where the stored strain energy, available for dissipation through an instantaneous delamination effect, is relatively high.

The main concept has been already formulated and discussed on the classical elastic beam model (Holnicki and Marzec, 1998) and a particular technical application has been proposed (Holnicki, 1997). The numerical simulation of the active damping process has been discussed by Marzec et al., 1998. Similar approach to the problem of strain energy dissipation, with use of controllable rotational dissipaters in joints of skeletal structures, has been also proposed recently (Gaul et al.,1998). The general problem formulation and the robustness of the impulse release strategy of the active damping of vibration will be demonstrated (in the final version of this paper) through numerical simulations as well as experimental verification. We assume that ideal actuators (dissipaters) can take one of the following two positions: a) the full connection (adhesion) in the controlled interface, b) the fully open position (delamination effect) when the controlled interface does not transfer any force.

The impulse release control strategy of dissipaters in the cantilever beam shown in Fig. 1 can be formulated as follows:

Instantaneous full opening of dissipaters (and full locking after a short time interval) whenever $\dot{\mathrm{u}}=0$ (extremal deflection of the beam) where $u$ denotes vertical movement of the node $A$.

In general, this strategy can be formulated locally, requiring instantaneous opening (for a short time interval) of dissipaters whenever local strain velocity drops to zero. 


\section{NUMERICAL EXAMPLES}

Let us demonstrate the effect of the strategy of the impulse release of strain energy for damping of free vibration on two examples of adaptive structures.

a) The free vibration of truss-beam structure shown in Fig.1 and exicited by the impulse force application to the tip point $\mathrm{A}$ has been demonstrated in Fig.2. (line uA shows oscilation of the tip point A). The structure response to the impulse release strategy of damping is shown in Fig.2 (line uAd), while the distribution of total kinetic (line Uk), strain (line Us) and dissipated (line Ud) energy in time is shown in Fig.3. Very high damping effect can be observed. The vibration is almost completely damped after two activations of dissipaters. The strain-stress histeretic loop for the chosen dissipater (No. 2) is shown in Fig.4.

b) The double layer beam Fig.5. with the third layer composed of switchable elements able to release instantly acumulated shear stresses can realise a controlled „delamination effect”. The free vibration response to the force impulse applied to the tip point $\mathrm{A}$ has been demonstrated in Fig. 6 (line $\mathrm{uA}$ ). While the structure response after application of the impulse release strategy of damping is shown in Fig. 6 (line uAd). The corresponding distribution of energies has been demonstrated in Fig. 7., while the strain-stress histeretic loop for interface element has been shown in Fig. 8.

The percentage of energy disspation effect (comparing with the initially introduced strain energy) during first four half-cycles (four activations of dissipaters) for two discussed above examples is shown in Table 1.

\section{Table 1. Energy Dissipation Effect}

\begin{tabular}{|l|c|c|}
\multicolumn{1}{c}{ Activations } & \multicolumn{1}{c|}{ Model a } & Model b \\
\hline 1 halfcycle & 52.2 & 16.0 \\
\hline 2 halfcycle & 69.5 & 57.5 \\
\hline 3 halfcycle & 70.0 & 72.0 \\
\hline 4 halfcycle & 72.0 & 75.2 \\
\hline
\end{tabular}

\section{EXPERIMENTAL TESTS}

Experimental verification of the influence of the controlled delamination effect on damping of free vibration has been performed using the double layer beam model (Figs. 9, 13) equipped with two, actively controlled electromagnetic clamping devices. These clampers can realise two functions: full adhesion between the two beam layers or full delamination effect. The active control process is reduced to the strategy of the clamper's switching on and off (Fig.10), where the time delay (426 $\mathrm{ms}$ after the registered extremal amplitude of the controlled first mode of free vibration) has been adjusted experimentally to maximise the overall damping effect. The time intervals for open clampers $(150 \mathrm{~ms})$ have been determined experimentally as the smallest interval required to realise full delamination effect. The experimental results for the uncontrolled free vibration of the fully clamped double layer beam and for the actively controlled beam have been shown in Figs 11 and 12, respectively. Two oscilating lines show deflection amplitudes of the points $\mathrm{x}_{1}$ and $\mathrm{x}_{2}$ (Fig.9) observed by two sensors. The formal time delay $426 \mathrm{~ms}$ for the halfcycle time $585 \mathrm{~ms}$ of vibration means that the advanced action $\mathrm{T}_{\text {advanced }}=159 \mathrm{~ms}$ of our actuators is applied in reality (cf. Fig.12 with the control strategy shown by the step function).

Despite of high natural damping of the tested model (cf. Fig.11), it is clearly seen the efficiency of the applied active damping strategy. The corresponding damping coefficient grows from 0.337 to 0.883 , respectively. In the further research a new experimental model will be presented. The heavy electromagnetic clampers disturbing the active control process will be replaced by the "amplified piezoelectric actuators" (cf. Bruneau et al., 1997) what will give us more reliable results, close to the ideal model with the controlled delamination effect. The calibrated numerical model of this tested experiment will be also elaborated. Then, farther improvements of the active damping control strategy will be discussed end numerically simulated. This simulation will include the strategy for active damping of vibration composed of the first two modes. In this case the following two possible strategies can be applied:

a) the local control strategy (described above) switching actuators due to locally detected extremes of vibration amplitudes,

b) the global control strategy making use of the numerical model of the structure and performing (in real time) identification of the current aigen modes' composition. 


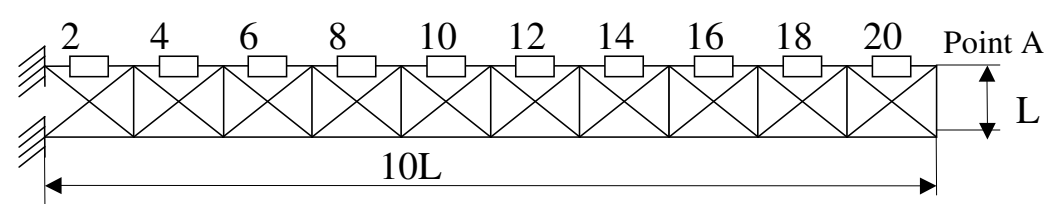

$\mathrm{E}_{\mathrm{i}}=200[\mathrm{GPa}]$

$\mathrm{A}_{\mathrm{i}}=0.0001\left[\mathrm{~m}^{2}\right]$

$\rho_{\mathrm{i}}=1 \mathrm{e}-6\left[\mathrm{~kg} / \mathrm{m}^{3}\right]$

$\mathrm{l}_{\mathrm{i}}=0,004$ [m.]

Fig. 1. Adaptive Truss Model

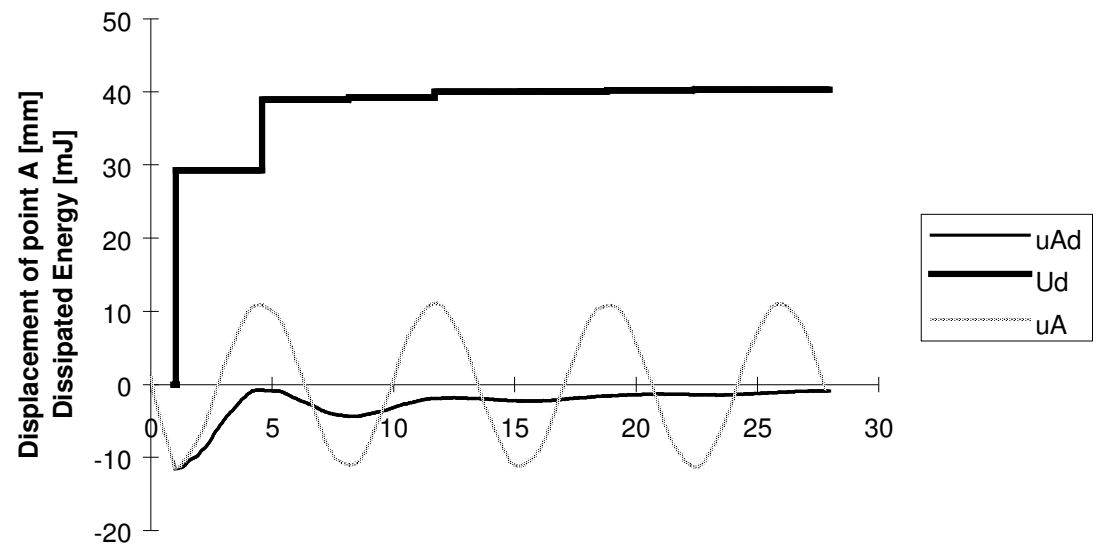

Time [s]

Fig. 2. Tip Point A Oscilations

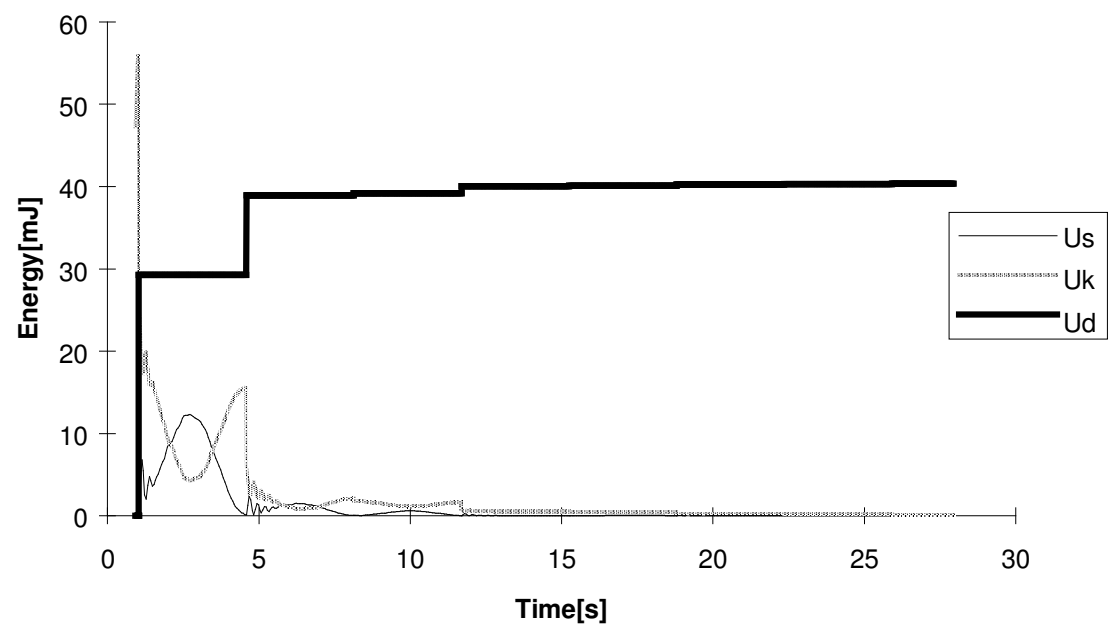

Fig. 3. Distribution of Energy Components

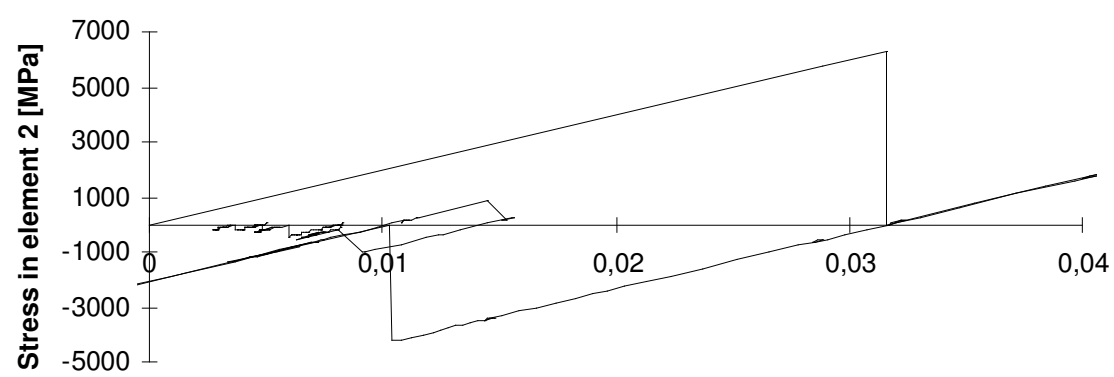

Strain in element $2[\mathrm{~mm} / \mathrm{mm}]$

Fig. 4. Histeretic Loops 


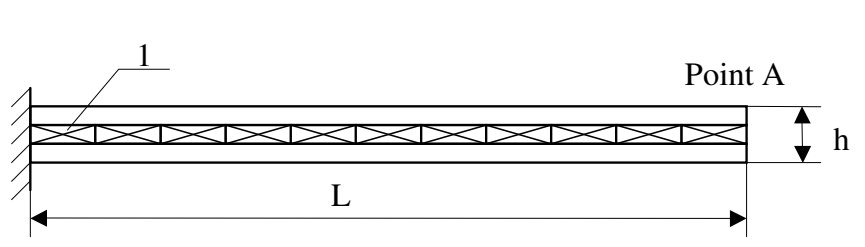

Fig. 5. Adaptive Double Layer Beam Model

$$
\begin{aligned}
& E=200[\mathrm{GPa}] \\
& v=0.3 \\
& \mathrm{l}=0.4[\mathrm{~m} .] \\
& \mathrm{h}=0.01[\mathrm{~m} .] \\
& d=0.01[\mathrm{~m} .] \\
& \rho=1 \mathrm{e}-6\left[\mathrm{~kg} / \mathrm{m}^{3}\right]
\end{aligned}
$$

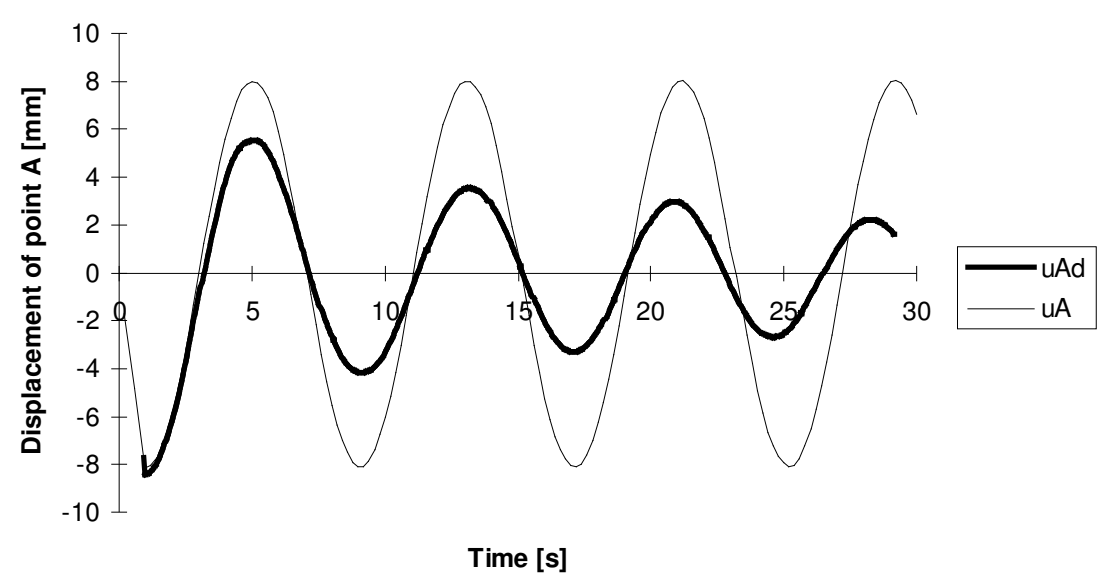

Fig. 6. Tip Point A Oscilations

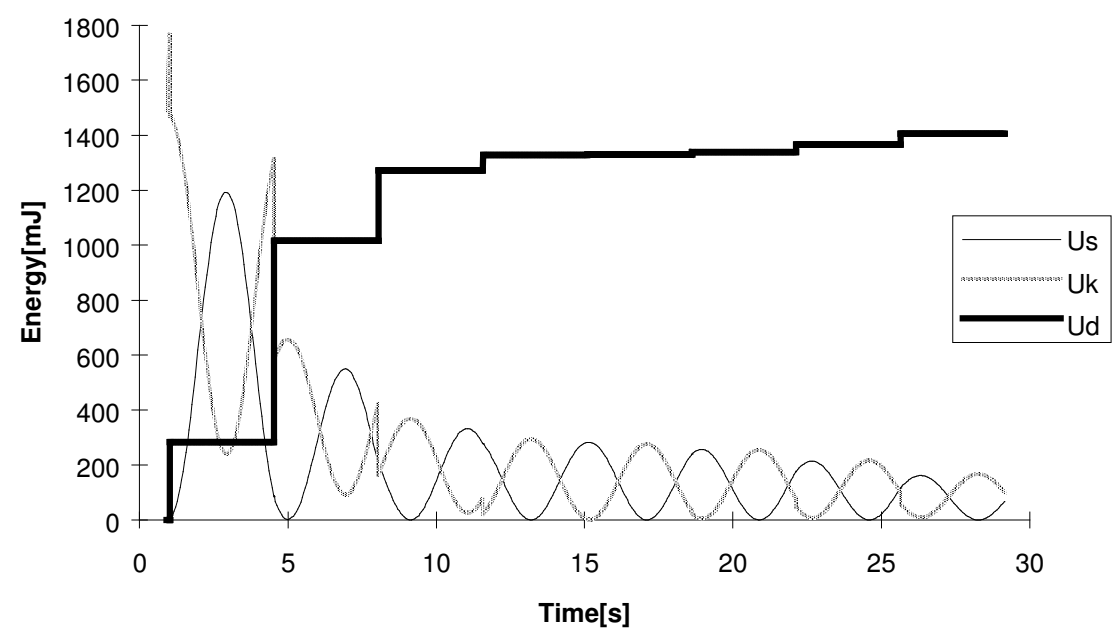

Fig. 7 Distribution of Energy Components

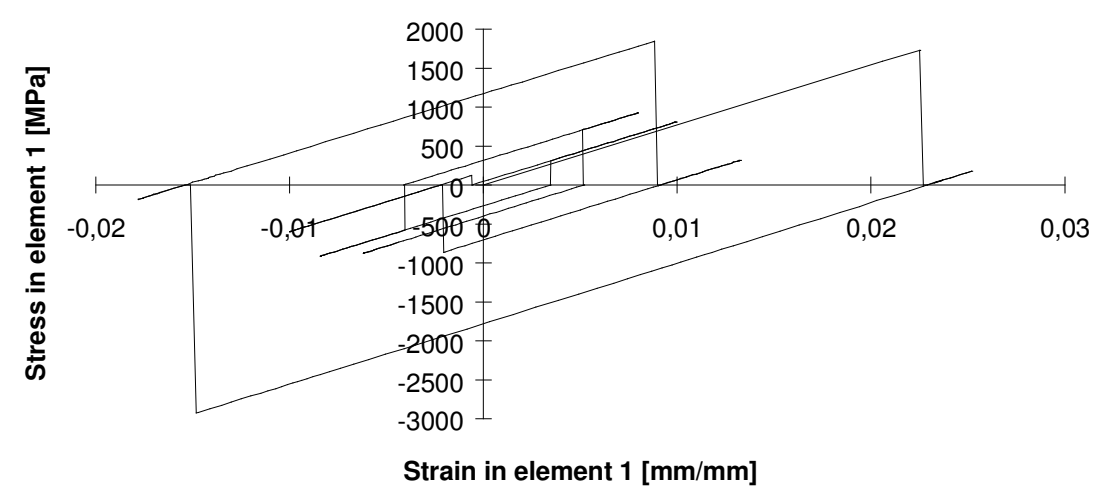

Fig. 8. Histeretic Loops 


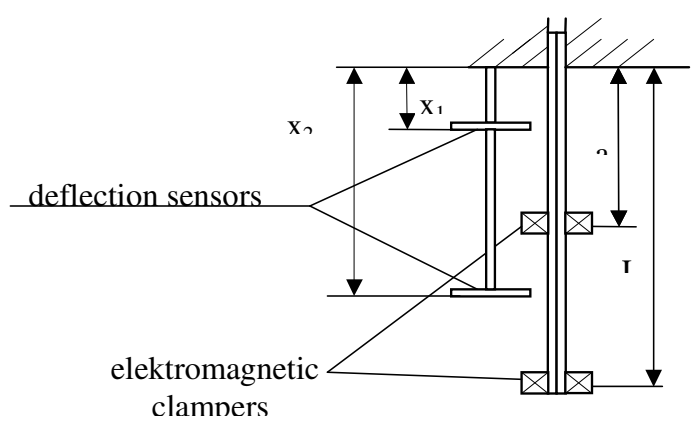

Fig.9 The double layer beam with actively controlled clampers

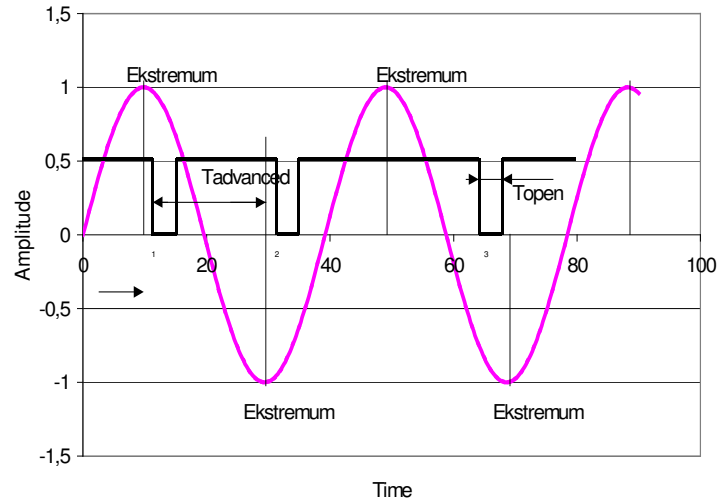

Fig.10 The active control strategy of the clamper's switching on and off

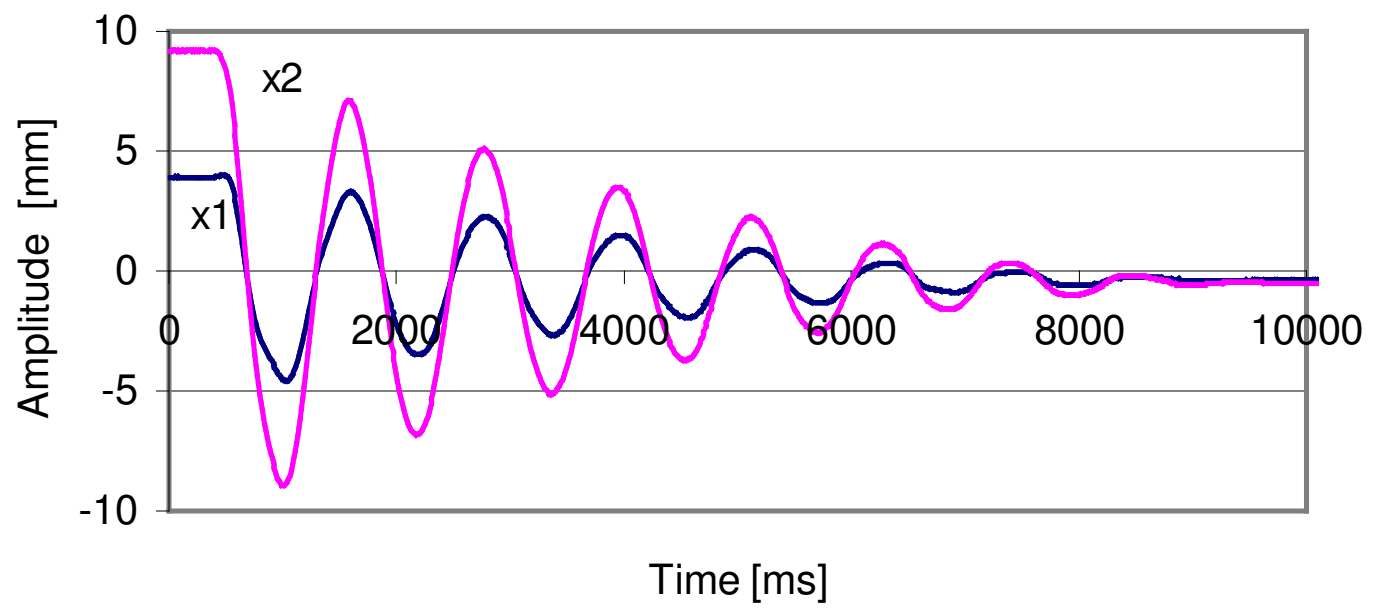

Fig.11 Free, uncontrolled vibration (the first mode) of the fully clamped double layer beam.

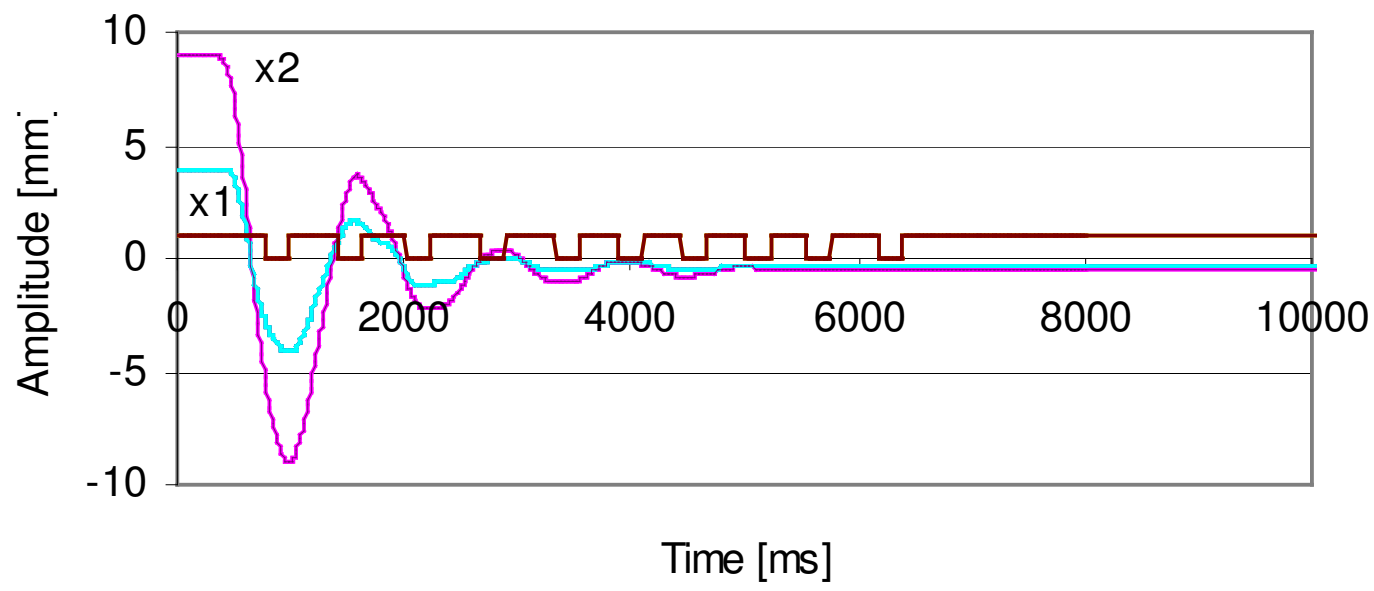

Fig.12 Actively damped vibration (the first mode) of the double layer beam and the applied control strategy 


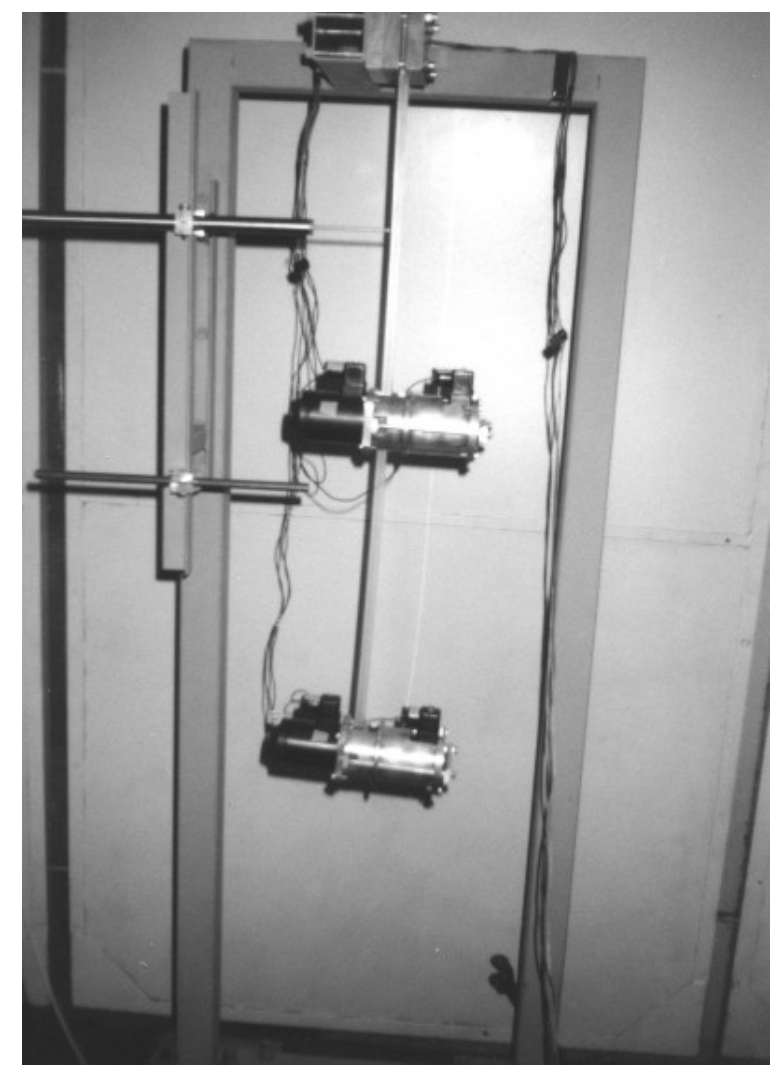

Fig.13 Double Layer Beam Model Assembly

\section{CONCLUSIONS}

High efficiency of the proposed semi-active vibration control has been demonstrated.

It has been shown that actively controlled, lowenergy consuming actuators, realising switchable behaviour of structural inter-connections allow effective vibration energy dissipation.

It has been demonstrated that double-layer beams (and similarly, plates and shells) with switchable delamination effect along the active interface are good examples of effectively controlled adaptive structures.

\section{ACKNOWLEDGEMENT}

The research presented in this paper was supported by the NATO Collaborative Research Grant No.OUTREACH CRG 950316 and by the NSF grant INT-9815060. This paper presents a part of the Ph.D. thesis of the second author, supervised by the first author.

\section{REFERENCES}

Holnicki-Szulc,J.andZ.Marzec,Adaptive Structures with Semi-Active Interface, Proc. of the EUROMECH 373 Colloquium on Modelling and Control of Adaptive Mechanical Structures, March 1998, Magdeburg

Marzec,Z, J.Holnicki-Szulc and F.Lopez-Almansa, Strategy of impulse release of strain energy for damping of vibration, Proc. NATO ARW Smart Structures'98, June 1998, Pultusk Poland

Holnicki-Szulc, J. Panel for Impact Energy Dissipation, Patent Licence applied for Warsaw, 1997

Gaul, L., R.Nitsche and D.Sachau, Semi-Active Vibration Control of Flexible Structures, Proc. of the EUROMECH 373 Colloquium on Modelling and Control of Adaptive Mechanical Structures, 1998, Magdeburg

Bruneau,H., R.Le Letty, F.Barillet, N.Lhermet, Application of a new amplified piezoelectric actuator tosemi-active control of vibration, Proc. $2^{\text {nd }}$ Int. Conference MV2 on Active Control in Mechanical Engineering, Lyon, 97 